\title{
Placing the RPL32 Promoter Upstream of a Second Promoter Results in a Strongly Increased Number of Stably Transfected Mammalian Cell Lines That Display High Protein Expression Levels
}

\author{
F. Hoeksema, K. Hamer, M. Siep, J. A. Verhees, and A. P. Otte \\ Swammerdam Institute for Life Sciences, University of Amsterdam, Science Park 904, 1098 XH Amsterdam, The Netherlands
}

Correspondence should be addressed to A. P. Otte, arie.otte@science.uva.nl

Received 8 September 2010; Accepted 4 November 2010

Academic Editor: Masaru Ohme-Takagi

Copyright ( 2011 F. Hoeksema et al. This is an open access article distributed under the Creative Commons Attribution License, which permits unrestricted use, distribution, and reproduction in any medium, provided the original work is properly cited.

\begin{abstract}
The use of high stringency selection systems commonly results in a strongly diminished number of stably transfected mammalian cell lines. Here we placed twelve different promoters upstream of an adjacent primary promoter and tested whether this might result in an increased number of colonies; this is in the context of a stringent selection system. We found that only the promoter of the human ribosomal protein, RPL32, induced a high number of colonies in CHO-DG44 cells. This phenomenon was observed when the RPL32 promoter was combined with the CMV, SV40, EF1- $\alpha$, and the $\beta$-actin promoters. In addition, these colonies displayed high protein expression levels. The RPL32 promoter had to be functionally intact, since the deletion of a small region upstream of the transcription start site demolished its positive action. We conclude that adding the RPL32 promoter to an expression cassette in cis may be a powerful tool to augment gene expression levels.
\end{abstract}

\section{Introduction}

Several approaches have been described that positively influence gene expression levels. One typical approach is to flank a gene expression cassette with DNA elements that somehow augment gene expression levels. Amongst these elements are MAR (Matrix Attachment Regions) elements [1-3], UCOEs (Ubiquitous Chromatin Opening Elements) $[4,5]$, insulators $[6,7]$, and STAR elements $[8]$. In varying degrees such elements convey higher protein expression levels in transfected mammalian cell cultures $[2,3,5,8]$. One approach is missing from this list: the use of a heterologous promoter that is combined with the promoter that normally drives gene expression in the expression cassette. In principle there is not much to say for such an approach. Placing two promoters adjacent to each other will often result in unwanted effects such as promoter interference, which refers to the direct negative impact of one transcriptional activity on a second transcriptional activity in cis [9]. Elaborate studies show the decline of promoter activities due to these phenomena [10-14]. However, some studies show a positive interaction among promoters. For instance, introducing an active promoter upstream of the silent human endogenous retrovirus (HERV)-K18 promoter activates its transcription in cis [15]. In addition, in case of integrated HIV-1 genomes within actively transcribed host genes in latently infected $\mathrm{CD}^{+} \mathrm{T}$ cells, read-through transcription-enhanced HIV-1 gene expression occurs when HIV-1 was in the same orientation as the host gene [16]. Furthermore, UCOEs are CpG island fragments containing two divergent promoters. When these elements are placed upstream of the hCMV promoter in mammalian cells, genomic locus silencing was reduced and transgene expression was enhanced [5].

Here we tested an array of twelve promoters and placed these immediately upstream of the human $\beta$-actin promoter. We did this in the context of a very stringent selection system for mammalian cells that we described previously. That system is based on the altered translation efficiency of the Zeocin-resistance selection marker [17-19]. For instance, the TTG Zeocin marker has a strongly impaired translation 
efficiency, as compared to the wild-type ATG Zeocin marker. For the cell to survive, sufficient Zeocin resistance protein is needed, and thus high levels of TTG Zeocin mRNA are needed to fulfil this requirement. When the TTG Zeocin gene is coupled to a gene of interest in such a way that a bicistronic mRNA is created, the high levels of this bicistronic mRNA will automatically result in high levels of the protein of interest. Importantly, DNA elements that augment gene expression are needed to induce such high mRNA levels [20]. Without DNA elements such as STARs, Zeocin gene expression is simply not high enough and no colonies are formed as a result. This creates a very low background, which makes it easy to assess the (positive) influences of exogenous factors on the number of induced colonies.

Of the twelve tested heterologous promoters, we found that one promoter, the human RPL32 promoter, was able to induce a high number of stably transfected colonies in CHO-DG44 cells. The endogenous RPL32 promoter drives the expression of the large ribosomal protein RPL32. The number of induced colonies with the RPL32- $\beta$-actin promoter combination was higher than with the addition of STAR elements that also strongly induced an increased number of colonies. We found that also the RPL32-CMV promoter combination resulted in a strong increase of induced colonies. Furthermore, the protein expression levels in these colonies were at least as high as in colonies induced with the aid of STAR elements. We finally show that the positive effects on colony formation and protein expression levels occur under different cell culturing conditions, such as serum free suspension culture medium. The addition of the heterologous RPL32 promoter to a gene expression cassette may be a powerful tool for augmenting gene expression levels.

\section{Results}

2.1. Experimental Setup. We tested whether a second promoter, placed upstream of the "primary" human $\beta$-actin promoter might have a positive influence on the number of colonies that are formed in the context of a very stringent selection system. Twelve promoters were chosen for testing: the viral CMV and SV40 promoters, the human $\beta$-actin, $\gamma$-actin $[21,22]$, ubiquitin C (UBC) [23], EF1- $\alpha$ [24], glyceraldehyde-3-phosphate dehydrogenase (GAPDH) [25], and five ribosomal protein (RPL32, RPLP1, RPS21, RPL8, and RPL41) promoters [26]. These human genes encode proteins that are, respectively, involved in cytoskeleton formation, protein degradation, protein biosynthesis, carbohydrate metabolism, and the formation of ribosomal subunits. As such they encode the so-called housekeeping proteins, proteins that are required for the maintenance of basal cellular functions $[27,28]$. The promoters that we selected belong to genes that convey high mRNA expression levels of their respective genes in human and mouse samples, as determined by Su et al. [29]. The promoters were isolated by PCR (see sequence listing for the primers) with human genomic DNA as template. The identity of the promoters was verified by DNA sequencing.
The promoters were cloned in the STAR-Select vector that conveys a high stringency of selection pressure [19] (Figure 1). In a STAR-Select vector, the Zeocin selection marker is modified to use a start codon that confers attenuated translation initiation frequency, such as TTG, instead of ATG. This modified selection marker is placed upstream of the d2EGFP reporter gene, which, in contrast, has a start codon that confers optimal translation initiation (Figure 1). The stringency of this selection system is so high that the human $\beta$-actin promoter is not strong enough to induce substantial colony formation (Figure 1). In fact, only when STAR elements are added to the construct, is the expression of the selection marker enough to induce colony formation (Figure 1) [19]. In these experiments, we therefore included the constructs without any elements as negative control and the construct flanked with the STAR 7/67/7 combination as positive control (Figure 1).

\subsection{Placing the Human RPL32 Promoter Upstream of the} Human $\beta$-Actin Promoter Results in the Induction of Many Colonies and High Protein Expression Levels. The same amount of DNA $(3 \mu \mathrm{g})$ of all constructs was transfected to CHO-DG44 cells with Lipofectamine 2000 (Invitrogen). Selection was performed with $400 \mu \mathrm{g} / \mathrm{ml}$ Zeocin in the culture medium, which was added 24 hours after transfection. After approximately two weeks, the number of stably established colonies was counted. As shown in Figure 2, transfection of the construct encompassing STAR 7/67/7 resulted in 125 stable colonies. The construct with only the human $\beta$-actin promoter (negative control) gave $<10$ colonies. A similar result ( $<25$ colonies) was obtained with ten heterologous promoter elements placed upstream of the human $\beta$-actin promoter, except for the construct with the RPL8 upstream of the human $\beta$-actin promoter (50 colonies), and in particular with the RPL32 promoter $(>400$ colonies) (Figure 1).

Up to 24 independent colonies induced by the indicated constructs were isolated. Colonies were propagated before analysis by flow cytometry (FACS), approximately 6 weeks after transfection. The fluorescence signal derived from d2EGFP (destabilized) is positively correlated with the promoter activity and is thus a reliable indicator of the d2EGFP expression levels in the cell. In a single FACS analysis, fluorescence signals from a sample that contains up to 4000 cells are analyzed. One such sample of cells is taken from an independent, stably transfected cell colony. Since the signal will vary amongst the individual cells in the colony, the mean fluorescence level of the $\sim 4000$ cells in the sample is taken as a measure for the d2EGFP expression level (arbitrary units) in the stably transfected cell colony. As shown in Figure 2, placing heterologous promoters upstream of the $\beta$-actin promoter resulted in varying $\mathrm{d} 2 \mathrm{EGFP}$ expression values. At the lower end of the spectrum, the EF1- $\alpha$ promoter, placed upstream of the $\beta$-actin promoter induced very low d2EGFP values (Figure 2), as well as a low number of colonies (Figure 1). In contrast, the human RPLP1 promoter, placed upstream of the $\beta$-actin promoter induced a small number of colonies (Figure 1), but the d2EGFP expression 

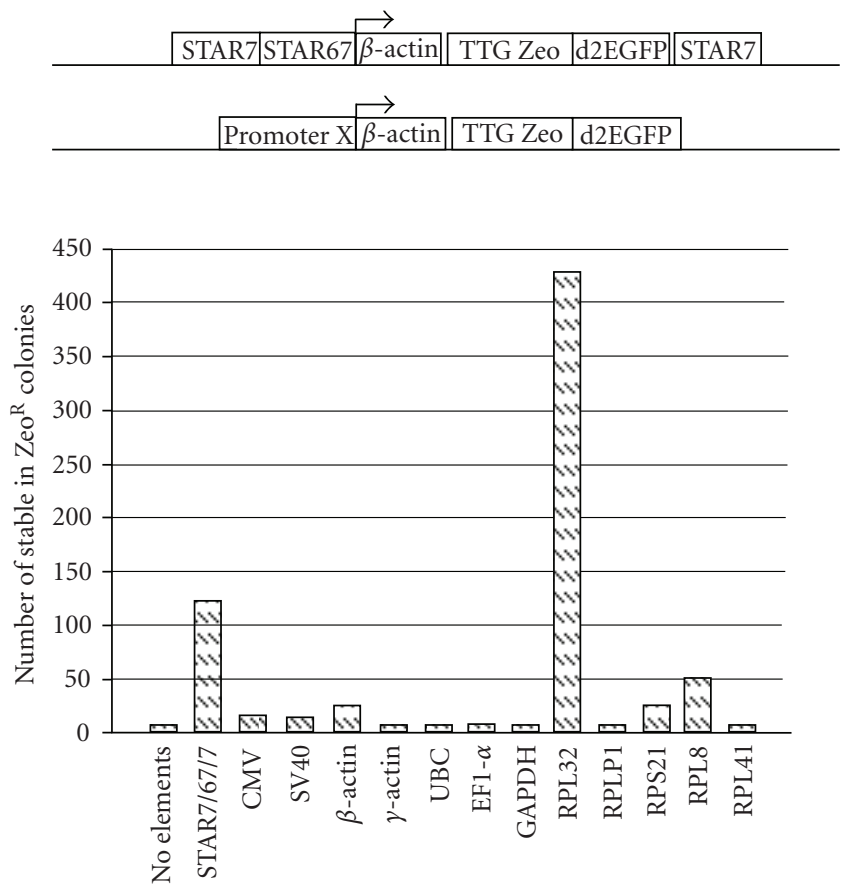

FIGURE 1: Heterologous promoters that are screened for their ability to elevate the formation of stable CHO-DG44 colonies in the context of a stringent selection system. Twelve human promoters were placed upstream of the human $\beta$-actin promoter. As selection system, a modified Zeocin selection marker, TTG Zeocin was placed upstream of the d2EGFP reporter gene. As control constructs, either no DNA elements are incorporated to the construct, or STAR elements are added to flank the expression cassette. Equal amounts of DNA were transfected to CHO-DG44 and after $\sim$ two weeks colonies were counted.

levels were high in these colonies (Figure 2). Importantly though, the d2EGFP expression levels in the construct with the RPL32- $\beta$-actin promoter combination were equally high as in the STAR 7/67/7-induced d2EGFP values (Figure 2).

We conclude that placing the RPL32 upstream of the $\beta$ actin promoter, in combination with the TTG Zeo d2EGFP expression unit induces more colonies than the STAR 7/67/7 combination in CHO-DG44 cells. Furthermore, the d2EGFP expression values in these clones equal the expression levels in the STAR 7/67/7-induced clones. In addition, when compared to the construct without STAR elements, incorporation of the RPL32 promoter in the construct leads to the induction of many more colonies.

2.2.1. Placing the heterologous RPL32 Promoter Upstream of Other Promoters Than the $\beta$-Actin Promoter Also Results in the Induction of many Colonies and High Protein Expression levels. The above experiments were all performed in the context of the $\beta$-actin promoter, and thus the effects of the heterologous RPL32 promoter might be promoter specific. We therefore also tested whether RPL32 had a beneficial effect on promoters other than the human $\beta$-actin promoter. We placed the RPL32 promoter upstream of the viral CMV and SV40 and the EF1 $\alpha$ promoters (Figure 3). As controls, we flanked the CMV, SV40, and EF1 $\alpha$ driven constructs with the STAR 7/67/7 combination, or with no flanking DNA elements at all. As shown in Figure 3, we found that the RPL32 promoter placed upstream of any promoter induced many more colonies as compared to the constructs without flanking DNA elements. When compared to the constructs containing STAR elements, the RPL32-CMV promoter combination induced $\sim 3$ times more colonies than the STAR 7/67/7-CMV promoter combination. This is similar to the result with the $\beta$-actin promoter (Figure 3). The RPL32 promoter induced, respectively, an equal number or $\sim 1.5$ times more colonies when placed upstream of the SV40 or EF $1 \alpha$ promoter.

When the d2EGFP expression levels were determined in these respective clones, we found that the RPL32- $\beta$-actin and RPL32-EF $1 \alpha$ promoter combinations induced equal d2EGFP expression levels as the STAR 7/67/7 combination (Figure 3), as also observed above. However, with the CMV and SV40 promoters, the RPL32 promoter induced substantially higher d2EGFP expression levels than the STAR 7/67/7 combination (Figure 3). Furthermore, transient d2EGFP expression levels were determined 24 hours after transfection of these plasmids. In all cases, RPL32 combined with another promoter showed increased transient d2EGFP expression levels compared to either the STAR 7/67/7 combination or with no flanking elements (data not shown). This indicates that RPL32 induces an increased transcription rate. We therefore conclude that the RPL32 promoter, placed upstream of different promoters, has a beneficial effect on these promoters, in terms of transient transcription levels, induced colony numbers, and protein expression levels and also that the observed effects are not restricted to the $\beta$-actin promoter. 

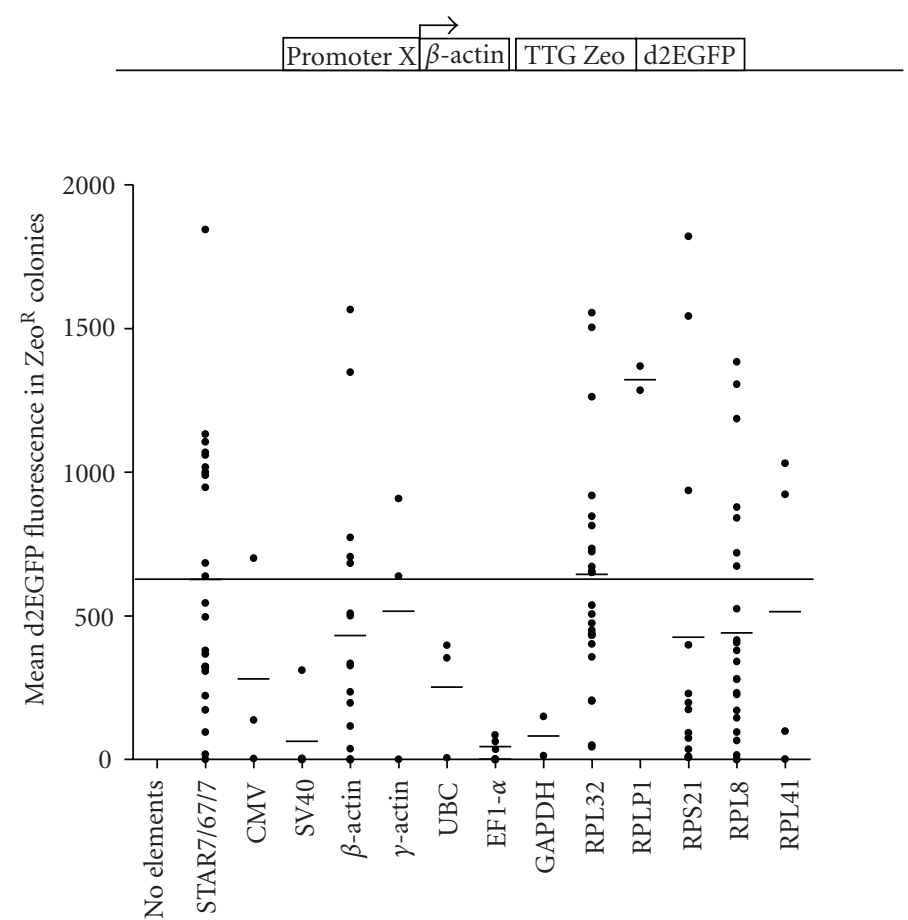

FIGURE 2: d2EGFP fluorescence values in colonies induced by heterologous promoter combinations. The d2EGFP expression values were determined in stable colonies comprising DNA constructs described in Figure 1. The relative fluorescence levels were taken as arbitrary units. The average d2EGFP expression levels for each construct are indicated with a short line. The average d2EGFP expression of 637 induced by STARs $7 / 67 / 7$ in the context of the TTG Zeo selection system is indicated with the horizontal line.

2.3. Requirements and Configurations That Influence the Positive Effects of the Heterologous RPL32 Promoter on Colony Formation and Protein Expression Levels. We created constructs in which the RPL32 promoter was placed in different orientations relative to the $\beta$-actin promoter, as shown in Figure 4. As control, we used the RPL32 promoter alone, placed immediately upstream of the TTG Zeo-d2EGFP cassette. As controls, we also used the $\beta$-actin promoter alone or the $\beta$-actin promoter with flanking STAR 7/67/7 elements. All plasmids were transfected to $\mathrm{CHO}-\mathrm{DG} 44$ cells, colonies were counted as described above, and the d2EGFP expression values in these colonies were determined. We noted that the RPL32 promoter alone induced more colonies than the $\beta$ actin promoter alone (Figure 4), in fact more than the range of the number of colonies that was induced by the CMV alone or the SV40 promoter alone (compare with Figure 3). However, either flanking STAR elements or the RPL32 promoter in combination with the $\beta$-actin promoter induced far more colonies (Figure 4). Specifically the construct, in which the single RPL32 promoter was placed upstream of the $\beta$-actin promoter, induced most colonies (424). Fewer colonies were induced when two RPL32 promoters were used to flank the entire construct on both sides (201). Finally, no colonies at all were induced when the orientation of the RPL32 was reversed, in the sense that the direction of transcription pointed away from the $\beta$-actin promoter in the construct (Figure 4 ). When the d2EGFP values were determined in the respective clones, we observed that the configuration in which a single RPL32 promoter was placed upstream of the $\beta$-actin promoter induced the highest average d2EGFP expression levels (Figure 4). Therefore, we favour the configuration in which the RPL32 promoter is placed head to tail with the $\beta$-actin promoter.

We next created a series of deletions of the RPL32 promoter to determine the potential crucial regions that convey the positive influence of the RPL32 promoter. As with all tested promoters, we initially isolated a rather large stretch of DNA surrounding the TATA boxes of the promoters. This was done, for instance, because these promoters show a high degree of homology among species in those regions (data not shown). However, the RPL32 promoter lacks a canonical TATA box. The so-called $\beta$ binding site of the RPL32 promoter contains a DNA sequence element (GGAA) and binds the transcription factor GA-binding protein (GABP), which is an Ets-related protein [30-33]. In spite of the lack of a canonical TATA element in the RPL32 gene promoter, TATA-binding protein (TBP) interacts with a region of 30 base pairs upstream of the cap site without the aid of other factors [34]. This region contains the binding site for the $\gamma$ factor and indicates that the $\gamma$ factor may play a role similar to TBP in transcription of the RPL32 gene.

As indicated in Figure 5(a), we deleted several portions, both $5^{\prime}$ and $3^{\prime}$ of the originally isolated $3220 \mathrm{bp}(-1918$ to +1302 bp with the transcription start at +1 ) RPL32 promoter 

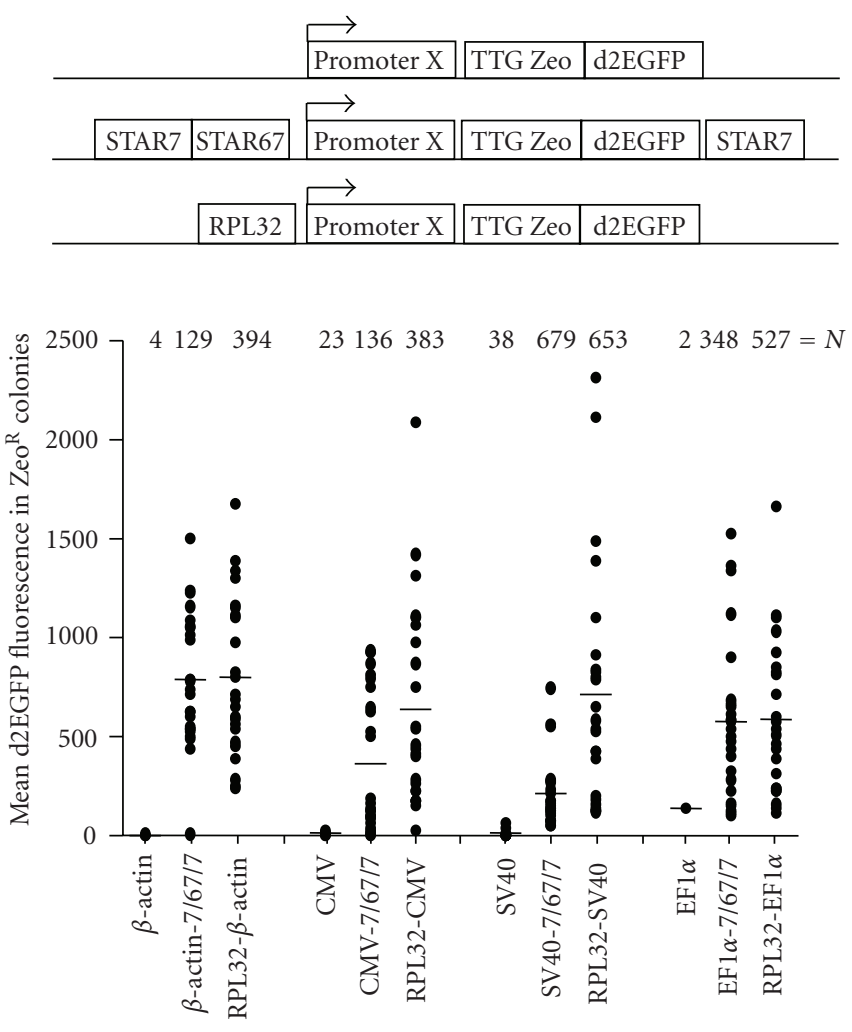

FIGURE 3: Addition of the RPL32 promoter has beneficial effects in the context of multiple heterologous promoters. The RPL32 promoter was placed upstream of the $\beta$-actin, CMV, SV40, and EF- $1 \alpha$ promoter, which drove the TTG Zeo- d2EGFP expression cassette. Control constructs with the four different promoters contained either no extra DNA element, or flanking STAR 7/67/7 elements. Equal amounts of DNA were transfected to CHO-DG44, and after approximately two weeks, colonies were counted. Up to 24 stable colonies were isolated, and $\mathrm{d} 2 \mathrm{EGFP}$ expression values were determined. The respective colony numbers are shown above the d2EGFP expression values.

region. In addition, we deleted the $76 \mathrm{bp}$ immediately upstream of the transcription start site that contains all the above-described binding sites that are considered important (Figure 5(a)). We called this construct $-1918-\Delta-1302$ (Figure 5(b)). We transfected the respective constructs to CHO-DG44 cells. As shown in Figure 5, we found that the $76 \mathrm{bp}$ upstream of the transcription start site of RPL32 was essential for its influence on the $\beta$-actin promoter. No colonies were formed by the construct containing the $-1918-\Delta-1302$ RPL32. Deletion from either the $5^{\prime}$ or $3^{\prime}$ side showed a more complex picture. $5^{\prime}$ deletions up to $691 \mathrm{bp}$ showed no significant changes in colony numbers and the respective d2EGFP values (Figures 5(a) and 5(b)). However, further $5^{\prime}$ deletions, up to $137 \mathrm{bp}$ of the transcription start (Figure 5(a)) resulted in a strongly decreased number of induced colonies and in the respective d2EGFP expression levels (Figure 5(b)). Deletions on the 3 ' side were even more complex. The deletion starting at $504 \mathrm{bp}$ downstream of the transcription start (construct -1918 to +504 ) resulted in increased colony numbers and d2EGFP expression levels equal to the full length RPL32. Instead, deletions starting at $94 \mathrm{bp}$ downstream of the transcription start both resulted in a large decrease of colony numbers and d2EGFP expression values (Figures 5(a) and 5(b)). When either part of the $5^{\prime}$ or the 3 ' side was deleted, both colony numbers and d2EGFP expression values decreased.
We conclude that a transcriptionally active RPL32 promoter is essential for its beneficial influence on the $\beta$-actin promoter and that the orientation of the RPL32 promoter must be that RPL32 transcription is in the same direction as that of the $\beta$-actin promoter (head to tail). Finally, the RPL32 variant in which the most $3^{\prime} \sim 800$ bp of the 3220 promoter region that we chose to isolate was deleted shows the most positive effects on the $\beta$-actin promoter, in terms of induced colony numbers.

2.4. The Positive Effects of the RPL32 Are Also Apparent in Suspension Cell Cultures. The above-described experiments were all performed in adherent cell cultures. It is, however, possible that promoters and gene-activity-enhancing elements operate differently under serum-free culturing conditions. We therefore transfected control and RPL32containing constructs under serum-free conditions to $\mathrm{CHO}-$ DG44-S suspension cells and cultured them likewise under serum-free suspension conditions. Cells were transfected (nucleofected) in chemically defined medium for DG44 suspension cells, with an Amaxa Nucleofector, and Zeocin selection was started. Three weeks after nucleofection, a stably transfected polyclonal population was poured in semisolid medium, for subcloning. Single colonies were isolated, transferred to 24-well plates and finally to T25 culture flasks. After another 2-3 weeks, protein expression levels were measured. 

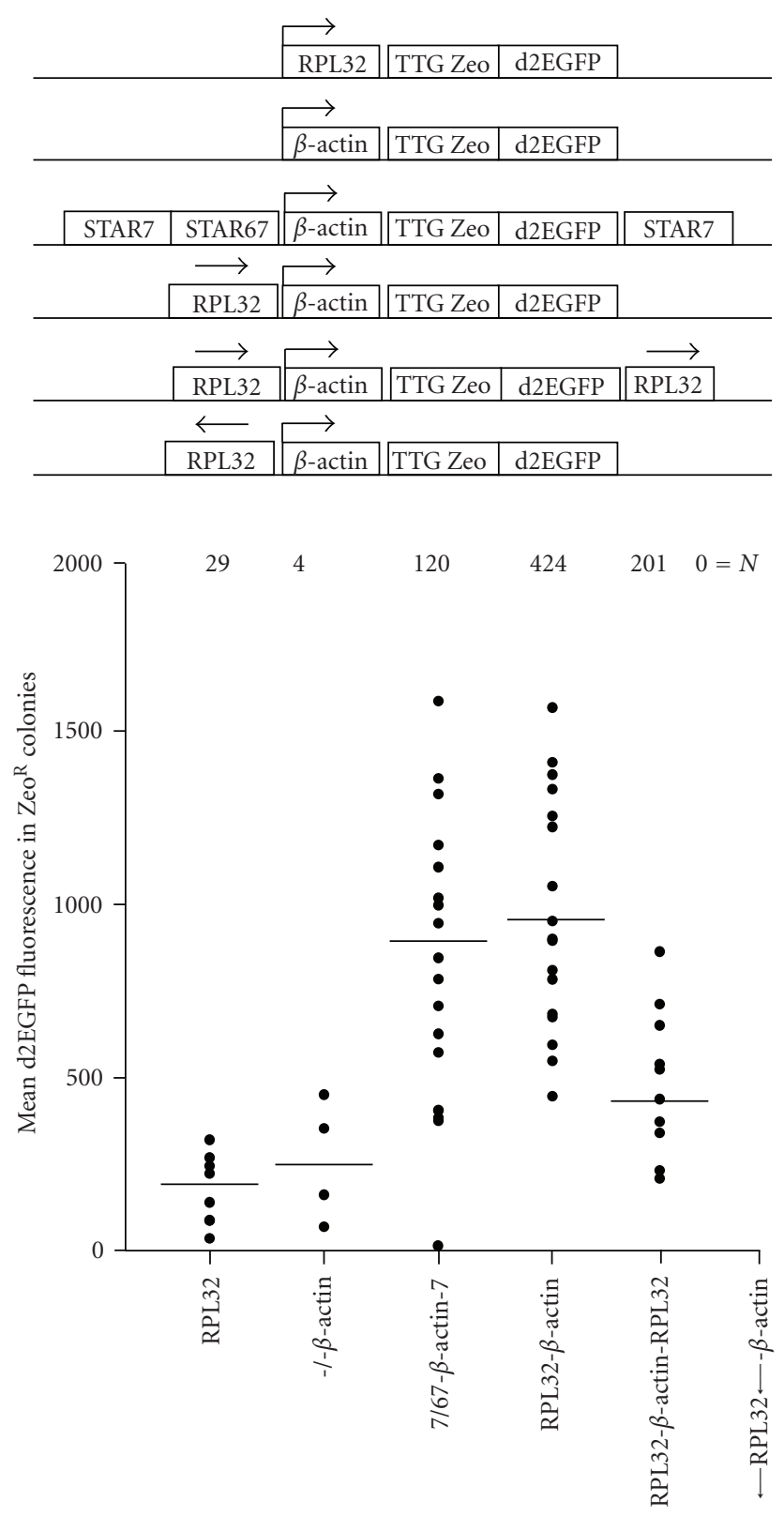

FIGURE 4: Optimal orientations of the RPL32 promoter in relation to the $\beta$-actin promoter. The RPL32 promoter was placed in configurations as shown at the top of the figure. Control constructs contained only the RPL32 promoter, the $\beta$-actin promoter without any DNA element, or with flanking STAR 7/67/7 elements. Equal amounts of DNA were transfected to CHO-DG44 and after approximately two weeks colonies were counted. Up to 24 stable colonies were isolated, and d2EGFP expression values were determined. The respective colony numbers are shown above the d2EGFP expression values.

The CMV promoter drove the expression units, either alone, or flanked by the STAR 7/67/7 combination (Figure 6). In another construct, the RPL32 promoter was placed upstream of the CMV promoter. Finally, a control construct consisted of the RPL32 promoter alone, without CMV promoter or other elements. Unlike adherent cell cultures, be counted independently established stable colonies in suspension cultures cannot. Therefore, as measure for the effectiveness of the different constructs, we determined the percentage of green fluorescent cells within the stably transfected polyclonal population, two weeks after nucleofection. As shown in Figure 6, after two weeks, the CMV promoter alone displayed only $7 \%$ green cells, and so did the RPL32 as promoter alone $(8 \%)$. Only when elements were added to the construct, did the percentage of stably transfected green cells increase significantly, two weeks after nucleofection. This indicated the beneficial effect of the RPL32 promoter on the growth of stably transfected cells. With flanking STAR elements the percentage rose to $15 \%$ cells and with the RPL32 promoter, placed upstream of the CMV promoter, to $35 \%$. The d2EGFP values were also determined in isolated subclones, which were cultured in T25 flasks for 2-3 weeks, as the d2EGFP expression values.

We noticed that the average d2EGFP expression values with the different constructs followed the same pattern as the percentage green cells in the polyclonal cell population, after two weeks. That is, the d2EGFP expression values were low with the CMV or RPL32 promoters alone (Figure 6), increased with flanking STAR elements, and were highest with the RPL32 placed upstream of the CMV promoter. These results show that also in suspension growing $\mathrm{CHO}-$ DG44-S cells, the RPL32 promoter is an effective tool to increase the number of gene expressing cells as well.

\section{Discussion}

In this paper, we describe the positive effects on recombinant mammalian cell line formation by placing the RPL32 promoter head-to-tail adjacent to a heterologous promoter. There are many papers describing the negative effects on transcription after placing two promoters adjacent to each other. In most cases, this results in transcriptional interference, which is interpreted as a negative effect [9]. Therefore, the results we showed came as rather unexpected. We conducted these experiments in course of a larger screening effort to identify DNA elements that are able to augment gene activity in the context of a stringent selection system for mammalian cells. Previously, we identified the so-called STAR elements that are able to induce more adherently growing colonies, when employed in a stringent selection system. Still, the number of adherent colonies induced by the presence of STAR elements is limited, and when CHO-DG44-S suspension cells are transfected, there are hardly any stably transfected suspension cell lines established, even when STAR elements are present in the construct. Successful transfection to suspension cells depends in part on the initial percentage of stably transfected cells with sufficient Zeocin-resistance protein expression levels. Elevating this percentage increases the probability to establish stably transfected suspension-growing cell lines. As shown here, incorporation of the extra RPL32 upstream of another promoter indeed allows a more efficient establishment of stably transfected suspension-growing cell lines. 


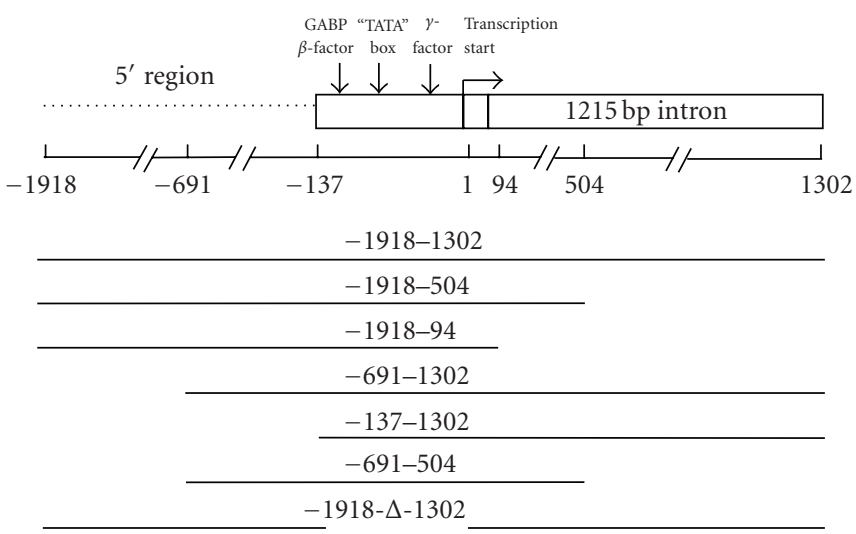

(a)

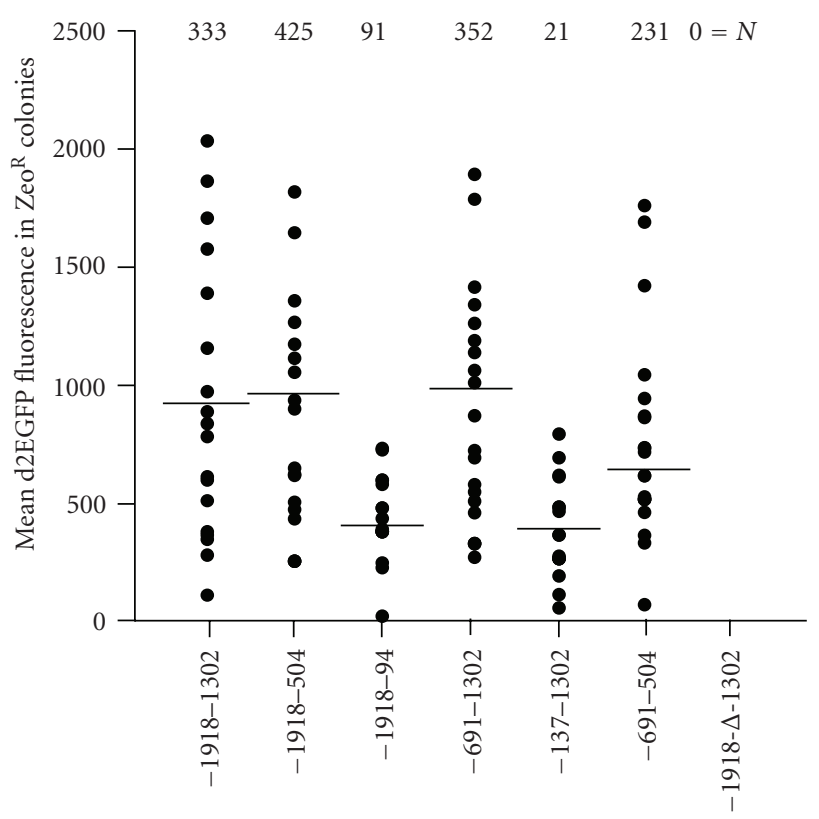

(b)

FIGURE 5: Deletion analysis of the RPL32 promoter reveals critical regions. Several deletion mutants of the RPL32 promoter were created as shown in (a). The constructs were transfected to CHO-DG44 cells, up to 24 stable colonies were isolated, and d2EGFP expression values were determined. The respective colony numbers are shown above the d2EGFP expression values.

How does this finding relate to previous work? Closest comes the work on UCOEs, which consist of CpG islands with two promoters that are placed in a natural occurring divergent manner. When this DNA element containing two promoters is placed upstream of a third promoter, this results in the creation of many more cell lines [5]. However, we previously showed that this does not result in higher protein expression levels, when employed in the context of the stringent selection system we use here [20]. Another approach has been to combine for instance the $\beta$-actin promoter with the CMV enhancer, leading to the creation of a hybrid promoter system. Also this has been reported to have a beneficial effect on protein expression levels [35]. Our results are different from this approach as well, in the sense that we utilize an entire promoter. As shown, the promoter has to be intact, since the deletion of a small region that encompasses the "core" RPL32 promoter region results in the complete loss of positive effects on the human $\beta$-actin promoter. This result alone makes it unlikely that RPL32 is an enhancer that is causal for the effects we observe.

The positive effects of addition of the RPL32 promoter we observe are not restricted to just one downstream promoter, but we found the positive effects in combination with the CMV, SV40, EF1- $\alpha$, and $\beta$-actin promoters. Although the effects on these different promoters showed subtle differences, they had the following in common. When compared to a construct that contained no additional DNA elements (such as STAR elements), both the numbers of induced colonies and the protein expression levels in these colonies were highly elevated, but why is it that only the RPL32 promoter, out of twelve tested promoters, has this effect?
It is possible that there is a coincidental combination of transcription factor binding sites in the RPL32 promoter and a second, downstream-located promoter that mediates this effect. However, occupation of one promoter by RNA polymerase II could preclude its occupation of the second promoter. For instance, transcription across a promoter from an external promoter transiently precludes its occupation by RNA polymerase II and possibly also associated transcription factors. Furthermore, promoters can compete for the same enhancer. Those are explanations that have been offered for negative promoter interference, and they could possibly also explain why the other eleven promoters we tested donot show positive effects. This is all, however, highly speculative and we do not know why it is that only the tested RPL32 promoter displays these positive effects.

We do know, however, some of the requirements that are needed for the RPL32 to operate in the described manner. For instance, we noticed that in transient transfections there is an increased transcription rate, as signified by the increased transient d2EGFP expression levels. These increases are in comparison with either no DNA elements or the flanking STAR elements in the construct. Also, reversal of the RPL32 promoter orientation in relation to the second promoter completely abolishes the positive effect on colony formation. Finally, deletion of putative regulatory sites in the "core" promoter region completely demolishes the positive effects of the RPL32 promoter. Taken together, we take these observations to support the notion that active transcription from the RPL32 promoter, in the same direction as the downstream promoter is needed to raise the transcriptional activity of downstream-located promoters. This elevated 


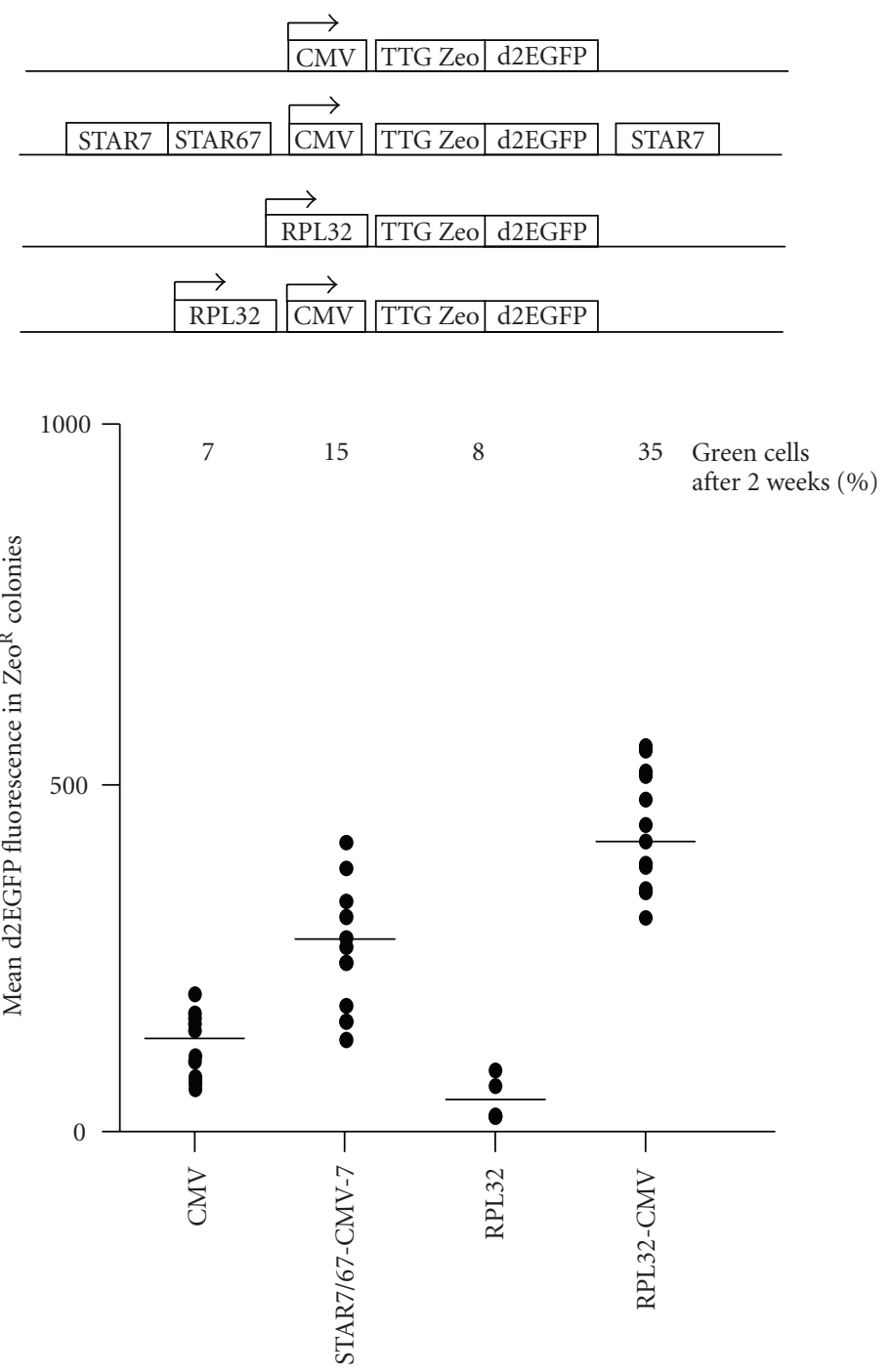

FIGURE 6: The beneficial effects of the RPL32 promoter on heterologous promoters are also observed in serum-free suspension cell lines. A construct in which the RPL32 promoter was placed upstream of the CMV promoter was compared to constructs with the CMV promoter, but without any DNA elements, or to a construct with STAR elements, as well a construct in which the RPL32 promoter alone drove the expression cassette. The constructs were transfected to serum-free suspension CHO-DG44-S cells. After two weeks, the percentage of green cells was determined, as shown in the numbers above d2EGFP values. Then subclones were isolated and propagated, and d2EGFP values were determined.

transcription rate is likely to raise the level of Zeocin selection marker protein, with as end result, an increased number of stably transfected colonies.

Deletion of a large portion of the $3^{\prime}$ located intron also brings the putative start site of the RPL32 start site closer to the start site of the human $\beta$-actin promoter. This results in an increased number of induced colonies, as compared to the use of the RPL32 that retains the entire intron. However, the putative start site of the RPL32 promoter is already very close to the start sites of the CMV (1890 bp), SV40 (1641 bp) or the EF1- $\alpha$ (1493 bp), promoters, as compared to the $3858 \mathrm{bp}$ of the human $\beta$-actin promoter transcription start site. Therefore, the distance between the start sites of the RPL32 and respective downstream promoters seems to be not very critical.
In conclusion, our results indicate that the RPL32 promoter can be a useful tool to increase the number of stably transfected colonies, when employed in the context of a stringent selection system. This may be in particular beneficial for suspension cells transfected and grown in serum-free suspension culture medium, which after all is the vehicle in which proteins are produced on an industrial scale.

\section{Experimental Protocol}

4.1. Vector Constructions. For cloning the various promoters, we used our STAR-Select vector [19] and first replaced the CMV promoter by either the human $\beta$-actin promoter, SV40 promoter, or EF $1 \alpha$ promoter. STAR elements 7 and $67\left(5^{\prime}\right)$ were replaced by the various promoters, and STAR 
$7\left(3^{\prime}\right)$ was removed. The human CMV immediate-early promoter/enhancer originated from pcDNA3 (Invitrogen) and the SV40 promoter from pBabe-Puro [36]. The other promoters were isolated by PCR using human genomic DNA as template. Primer sequences used for these PCRs are as follows (F: forward; R: reverse):

$\beta$-actin:

F: 5' GCCCCAGTGACAGCTCCGAAAGCTCCCTTACAGGGCAAAG 3

R: 5' GGTGAGCTGCGAGAATAGCCGGGCGCGCTG 3';

$\gamma$-actin promoter:

F: 5' AATTCCAGCAGCGCACAAGGAAACCGTAGTGC 3'

R: 5' TGCGACCTGCCCGGAAAAGGATGGACTCAG 3';

UBC promoter:

F: 5' CCATGCCTCCCTGTTGGCATCAAGTAGGACC $3^{\prime}$

R: 5' TGTCTAACAAAAAAGCCAAAAACGGCCAGAATTTAGCGGAC 3';

GAPDH promoter:

F: 5' CACAATGTCAATAGCGTCACAGTTGAGAAAACCTGC 3'

R: 5' GGTGTCTGAGCGATGTGGCTCGGC 3';

RPS21 promoter:

F: 5' TTTGAGACGCAGTCTTGCTCTGTCGCCCAGGCTGG 3'

R: $5^{\prime}$ TTCGAGGCTGGGCTGCGCCTGGGGAGTCAC 3';

RPL41 promoter:

F: 5' GAGGCGGGAGAATCGCTTGTATTCAGGAGG 3'

R: 5' GGCGCAGAGGTTTCTACAGGGAAAGAGAG 3';

RPL32 promoter:

F: 5' CTTGGCATTGACTTAGACACCCTAGGAATCTAACTTGAG 3'

R: 5' GATGCCTTTTGGGGAAGAAGCGGC 3';

RPL8 promoter:

F: 5' TGTGAGCAACAGCGGGCACAGGACACCCTTC $3^{\prime}$
R: 5' GGCGACGGGTCCTGGGGGCGACTCACGATTAG 3';

RPLP1 promoter:

F: 5' GGGGCAGTGGAATTTGTCTGAAGTAACTGTTGAATCCAC 3'

R: 5' GGCGCGGGCGAGTGTAGGGCTG 3';

EF1 $\alpha$ promoter:

F: 5' GTGCCCGTCAGTGGGCAG 3

R: 5' TCACGACACCTGAAATGGAAG 3';

RPL32 (-1918-504):

F: 5' CTTGGCATTGACTTAGACACCCTAGGAATCTAACTTGAG $3^{\prime}$

R: 5' CGACCTACAGCTCGTCTTTCCTTGG 3';

RPL32 (-1918-94):

F: 5' CTTGGCATTGACTTAGACACCCTAGGAATCTAACTTGAG $3^{\prime}$

R: 5' GCCAGATGAATCCCGCAGGAATGC 3';

RPL32 (-691-1302):

F: 5 AGTAGCTCGTGCCCGTAATCCCAG 3

R: 5' GATGCCTTTTGGGGAAGAAGCGGC 3';

RPL32 (-137-1302):

F: 5' CTCAGAATTTTTGCGGCATTATTTTTTGACGTGTC 3'

R: 5' GATGCCTTTTGGGGAAGAAGCGGC 3';

RPL32 (-691-504):

F: 5' AGTAGCTCGTGCCCGTAATCCCAG 3

R: 5' CGACCTACAGCTCGTCTTTCCTTGG; 3'

RPL32 (-1918- $\triangle-1302):$

F: 5' CTTGGCATTGACTTAGACACCCTAGGAATCTAACTTGAG $3^{\prime}$

R: 5' GCTCCGGCTCTTTTAAATAAAATAAAGACACGTC 3

F: 5' CTCTTCCTCGGCGCTGCCTAC 3'

R: 5' GATGCCTTTTGGGGAAGAAGCGGC 3'.

For RPL32 (-1918- $\triangle-1302)$, two PCRs were performed to remove the region containing binding sites important for promoter functioning. The two PCR fragments were ligated by using a restriction site introduced by PCR. 
A short DNA sequence run was performed on the isolated DNA sequences to verify that we indeed isolated the intended sequences.

4.2. Cell Culture, Transfection, and Analysis of Clones. CHODG44 cells [37] were grown in HamF12:DMEM medium (Invitrogen), supplemented with $4.6 \%$ fetal bovine serum (FBS) (Invitrogen), $2 \mathrm{mM}$ glutamine (Invitrogen), $100 \mathrm{U} / \mathrm{ml}$ penicillin (Invitrogen), $100 \mu \mathrm{g} / \mathrm{ml}$ streptomycin (Invitrogen), $100 \mu \mathrm{M}$ sodium hypoxanthine (Invitrogen), $16 \mu \mathrm{M}$ thymidine (Invitrogen), and $10 \mathrm{mM} \mathrm{MgCl}_{2}$ at $37^{\circ} \mathrm{C} / 5 \% \mathrm{CO}_{2}$.

For transfections, $0.4 \cdot 10^{6} \mathrm{CHO}-\mathrm{DG} 44$ cells were seeded in 6-well culture plates 24 hours prior to transfection. Cells were transfected with $3 \mu \mathrm{g}$ of plasmid DNA using Lipofectamine 2000 (Invitrogen) as described by the manufacturer. In brief, Lipofectamine 2000 was combined with plasmid DNA at $4 \mu \mathrm{l} / \mu \mathrm{g}$ DNA. The mixture was added to the cells, which had grown to $70-90 \%$ confluence. After 5 hours, the transfection mixture was replaced by fresh medium. The following day, cells were seeded in serial dilutions into a medium containing Zeocin (Invitrogen) at a concentration of $400 \mu \mathrm{g} / \mathrm{ml}$. Approximately 12 days after transfection, individual colonies became visible, and these were isolated and propagated in 24-well plates in medium containing Zeocin. When grown to $\sim 70 \%$ confluence, cells were transferred to 6 well plates. Cells were continued to grow in 6-well plates for another one to two weeks before FACS analysis or ELISA was performed. The d2EGFP expression levels were determined on an Epics XL Beckman Coulter flow cytometer. In case of transient transfections, d2EGFP expression levels were determined 24 hours following transfection. Values were visualized using Graphpad Prism 5 for Windows.

Wild-type CHO DG44-S suspension cells (Invitrogen) were grown in serum-free CD-DG44 medium (Invitrogen) supplemented with $8 \mathrm{mM}$ glutamine (Invitrogen), pluronic acid (Invitrogen), and anticlumping agent (Invitrogen) at $37^{\circ} \mathrm{C} / 8 \% \mathrm{CO}_{2}$ on a shaker $(130 \mathrm{rpm})$. Cells were passaged every 2-3 days. Cells were transfected (nucleofected) with an Amaxa Nucleofector, using the Nucleofection-kit Amaxa V, as described by the manufacturer. In brief, culture medium was supplemented with ITS (Invitrogen), and medium was equilibrated in the incubator to adjust $\mathrm{pH}$. For each nucleofection, $1 \cdot 10^{6}$ wild-type DG44-S cells, grown to a density between $7 \cdot 10^{5}$ and $1 \cdot 10^{6} / \mathrm{ml}$ and with a viability of $>90 \%$, were centrifuged in a swing out centrifuge $(900 \mathrm{rpm}, 5$ minutes). Cell pellets were dissolved in $100 \mu \mathrm{l}$ nucleofector solution, and $5 \mu \mathrm{g}$ DNA (in a volume of $5 \mu \mathrm{l}$ ) was added. Samples were transferred to a cuvette and electroporation in the Amaxa Nucleofector was performed (using program U-30), after which the samples were transferred to the equilibrated culture medium (in 6-well culture plates). After 5 hours, the cells were transferred to T25 (suspension) culture flask, in a total volume of $5 \mathrm{ml}$. After 48 hours, selection was started by adding $50 \mu \mathrm{g} / \mathrm{ml}$ Zeocin to the culture medium. Medium was refreshed every 2-3 days. During the next three weeks, the viability of the cells was monitored and, if applicable, d2EGFP expression levels were determined. Three weeks after nucleofection, 5000-10000 viable cells/ml were poured in semisolid medium (Genetix), to form subclones. After ten days, colonies were isolated and transferred to 96well culture plates in $100 \mu \mathrm{l}$ culture medium. After another week, cells could be transferred to 24-well plates (in $0.5 \mathrm{ml}$ medium). Selected subclones were propagated to grow in T25 culture flasks (in $5 \mathrm{ml}$ medium). After another 2-3, weeks d2EGFP expression levels were determined.

\section{References}

[1] M. Zahn-Zabal, M. Kobr, P. A. Girod et al., "Development of stable cell lines for production or regulated expression using matrix attachment regions," Journal of Biotechnology, vol. 87, no. 1, pp. 29-42, 2001.

[2] J. M. Kim, J. S. Kim, D. H. Park et al., "Improved recombinant gene expression in $\mathrm{CHO}$ cells using matrix attachment regions," Journal of Biotechnology, vol. 107, no. 2, pp. 95-105, 2004.

[3] P. A. Girod, M. Zahn-Zabal, and N. Mermod, "Use of the chicken lysozyme 5 ' matrix attachment region to generate high producer $\mathrm{CHO}$ cell lines," Biotechnology and Bioengineering, vol. 91, no. 1, pp. 1-11, 2005.

[4] M. Antoniou, L. Harland, T. Mustoe et al., "Transgenes encompassing dual-promoter $\mathrm{CpG}$ islands from the human TBP and HNRPA2B1 loci are resistant to heterochromatinmediated silencing," Genomics, vol. 82, no. 3, pp. 269-279, 2003.

[5] S. Williams, T. Mustoe, T. Mulcahy et al., "CpG-island fragments from the HNRPA2B1/CBX3 genomic locus reduce silencing and enhance transgene expression from the hCMV promoter/enhancer in mammalian cells," BMC Biotechnology, vol. 5, article no. 17, 2005.

[6] T. I. Gerasimova and V. G. Corces, "Chromatin insulators and boundaries: effects on transcription and nuclear organization," Annual Review of Genetics, vol. 35, pp. 193-208, 2001.

[7] V. J. Mutskov, C. M. Farrell, P. A. Wade, A. P. Wolffe, and G. Felsenfeld, "The barrier function of an insulator couples high histone acetylation levels with specific protection of promoter DNA from methylation," Genes and Development, vol. 16, no. 12, pp. 1540-1554, 2002.

[8] T. H. J. Kwaks, P. Barnett, W. Hemrika et al., "Identification of anti-repressor elements that confer high and stable protein production in mammalian cells," Nature Biotechnology, vol. 21, no. 5, pp. 553-558, 2003.

[9] K. E. Shearwin, B. P. Callen, and J. B. Egan, "Transcriptional interference-a crash course," Trends in Genetics, vol. 21, no. 6, pp. 339-345, 2005.

[10] I. H. Greger, F. Demarchi, M. Giacca, and N. J. Proudfoot, "Transcriptional interference perturbs the binding of Sp1 to the HIV-1 promoter," Nucleic Acids Research, vol. 26, no. 5, pp. 1294-1300, 1998.

[11] P. Wang, J. Yang, A. Ishihama, and A. J. Pittard, "Demonstration that the TyrR protein and RNA polymerase complex formed at the divergent $\mathrm{P} 3$ promoter inhibits binding of RNA polymerase to the major promoter, $\mathrm{P} 1$, of the aroP gene of Escherichia coli," Journal of Bacteriology, vol. 180, no. 20, pp. 5466-5472, 1998.

[12] S. K. Eszterhas, E. E. Bouhassira, D. I. K. Martin, and S. Fiering, "Transcriptional interference by independently regulated genes occurs in any relative arrangement of the genes and is influenced by chromosomal integration position," Molecular and Cellular Biology, vol. 22, no. 2, pp. 469-479, 2002. 
[13] B. P. Callen, K. E. Shearwin, and J. B. Egan, "Transcriptional interference between convergent promoters caused by elongation over the promoter," Molecular Cell, vol. 14, no. 5, pp. 647656, 2004.

[14] A. Buetti-Dinh, R. Ungricht, J. Z. Kelemen, C. Shetty, P. Ratna, and A. Becskei, "Control and signal processing by transcriptional interference," Molecular Systems Biology, vol. 5, article no. 300, 2009.

[15] O. Leupin, C. Attanasio, S. Marguerat, M. Tapernoux, S. E. Antonarakis, and B. Conrad, "Transcriptional activation by bidirectional RNA polymerase II elongation over a silent promoter," EMBO Reports, vol. 6, no. 10, pp. 956-960, 2005.

[16] Y. Han, Y. B. Lin, W. An et al., "Orientation-dependent regulation of integrated HIV-1 expression by host gene transcriptional readthrough," Cell Host and Microbe, vol. 4, no. 2, pp. 134-146, 2008.

[17] M. Kozak, "Context effects and inefficient initiation at nonAUG codons in eucaryotic cell-free translation systems," Molecular and Cellular Biology, vol. 9, no. 11, pp. 5073-5080, 1989.

[18] M. Kozak, "Downstream secondary structure facilitates recognition of initiator codons by eukaryotic ribosomes," Proceedings of the National Academy of Sciences of the United States of America, vol. 87, no. 21, pp. 8301-8305, 1990.

[19] H. J. M. van Blokland, T. H. J. Kwaks, R. G. A. B. Sewalt et al., "A novel, high stringency selection system allows screening of few clones for high protein expression," Journal of Biotechnology, vol. 128, no. 2, pp. 237-245, 2007.

[20] A. P. Otte, T. H. J. Kwaks, R. J. M. Van Blokland et al., "Various expression-augmenting DNA elements benefit from STARselect, a novel high stringency selection system for protein expression," Biotechnology Progress, vol. 23, no. 4, pp. 801-807, 2007.

[21] P. Ponte, S. Y. Ng, J. Engel, P. Gunning, and L. Kedes, "Evolutionary conservation in the untranslated regions of actin mRNAs: DNA sequence of a human beta-actin cDNA," Nucleic Acids Research, vol. 12, no. 3, pp. 1687-1696, 1984.

[22] H. P. Erba, P. Gunning, and L. Kedes, "Nucleotide sequence of the human $\gamma$ cytoskeletal actin mRNA: anomalous evolution of vertebrate non-muscle actin genes," Nucleic Acids Research, vol. 14, no. 13, pp. 5275-5294, 1986.

[23] O. Wiborg, M. S. Pedersen, A. Wind, L. E. Berglund, K. A. Marcker, and J. Vuust, "The human ubiquitin multigene family: some genes contain multiple directly repeated ubiquitin coding sequences," EMBO Journal, vol. 4, no. 3, pp. 755-759, 1985.

[24] T. Uetsuki, A. Naito, S. Nagata, and Y. Kaziro, "Isolation and characterization of the human chromosomal gene for polypeptide chain elongation factor- $1 \alpha$," Journal of Biological Chemistry, vol. 264, no. 10, pp. 5791-5798, 1989.

[25] L. Ercolani, B. Florence, M. Denaro, and M. Alexander, "Isolation and complete sequence of a functional human glyceraldehyde-3-phosphate dehydrogenase gene," Journal of Biological Chemistry, vol. 263, no. 30, pp. 15335-15341, 1988.

[26] R. P. Perry, "The architecture of mammalian ribosomal protein promoters," BMC Evolutionary Biology, vol. 5, 2005.

[27] J. A. Warrington, A. Nair, M. Mahadevappa, and M. Tsyganskaya, "Comparison of human adult and fetal expression and identification of 535 housekeeping/maintenance genes," Physiological Genomics, vol. 2000, no. 2, pp. 143-147, 2000.

[28] E. Eisenberg and E. Y. Levanon, "Human housekeeping genes are compact," Trends in Genetics, vol. 19, no. 7, pp. 362-365, 2003.
[29] A. I. Su, M. P. Cooke, K. A. Ching et al., "Large-scale analysis of the human and mouse transcriptomes," Proceedings of the National Academy of Sciences of the United States of America, vol. 99, no. 7, pp. 4465-4470, 2002.

[30] C. C. Thompson, T. A. Brown, and S. L. McKnight, "Convergence of Ets- and Notch-related structural motifs in a heteromeric DNA binding complex," Science, vol. 253, no. 5021, pp. 762-768, 1991.

[31] K. Macleod, D. Leprince, and D. Stehelin, "The ets gene family," Trends in Biochemical Sciences, vol. 17, no. 7, pp. 251256, 1992.

[32] T. Yoganathan, N. K. Bhat, and B. H. Sells, "A positive regulator of the ribosomal protein gene, $\beta$ factor, belongs to the ETS oncoprotein family," Biochemical Journal, vol. 287, no. 2, pp. 349-353, 1992.

[33] R. R. Genuario, D. E. Kelley, and R. P. Perry, "Comparative utilization of transcription factor GABP by the promoters of ribosomal protein genes rpL30 and rpL32," Gene Expression, vol. 3, no. 3, pp. 279-288, 1993.

[34] T. Yoganathan, M. Horikoshi, S. Hasegawa, R. G. Roeder, and B. H. Sells, "Yeast transcription factor IID participates in cellfree transcription of a mammalian ribosomal protein TATAless promoter," Biochemical Journal, vol. 285, no. 3, pp. 721723, 1992.

[35] S. Chung, T. Andersson, K. C. Sonntag, L. Björklund, O. Isacson, and K. S. Kim, "Analysis of different promoter systems for efficient transgene expression in mouse embryonic stem cell lines," Stem Cells, vol. 20, no. 2, pp. 139-145, 2002.

[36] J. P. Morgenstern and H. Land, "Advanced mammalian gene transfer: high titre retroviral vectors with multiple drug selection markers and a complementary helper-free packaging cell line," Nucleic Acids Research, vol. 18, no. 12, pp. 3587-3596, 1990.

[37] G. Urlaub, E. Kas, A. M. Carothers, and L. A. Chasin, "Deletion of the diploid dihydrofolate reductase locus from cultured mammalian cells," Cell, vol. 33, no. 2, pp. 405-412, 1983. 

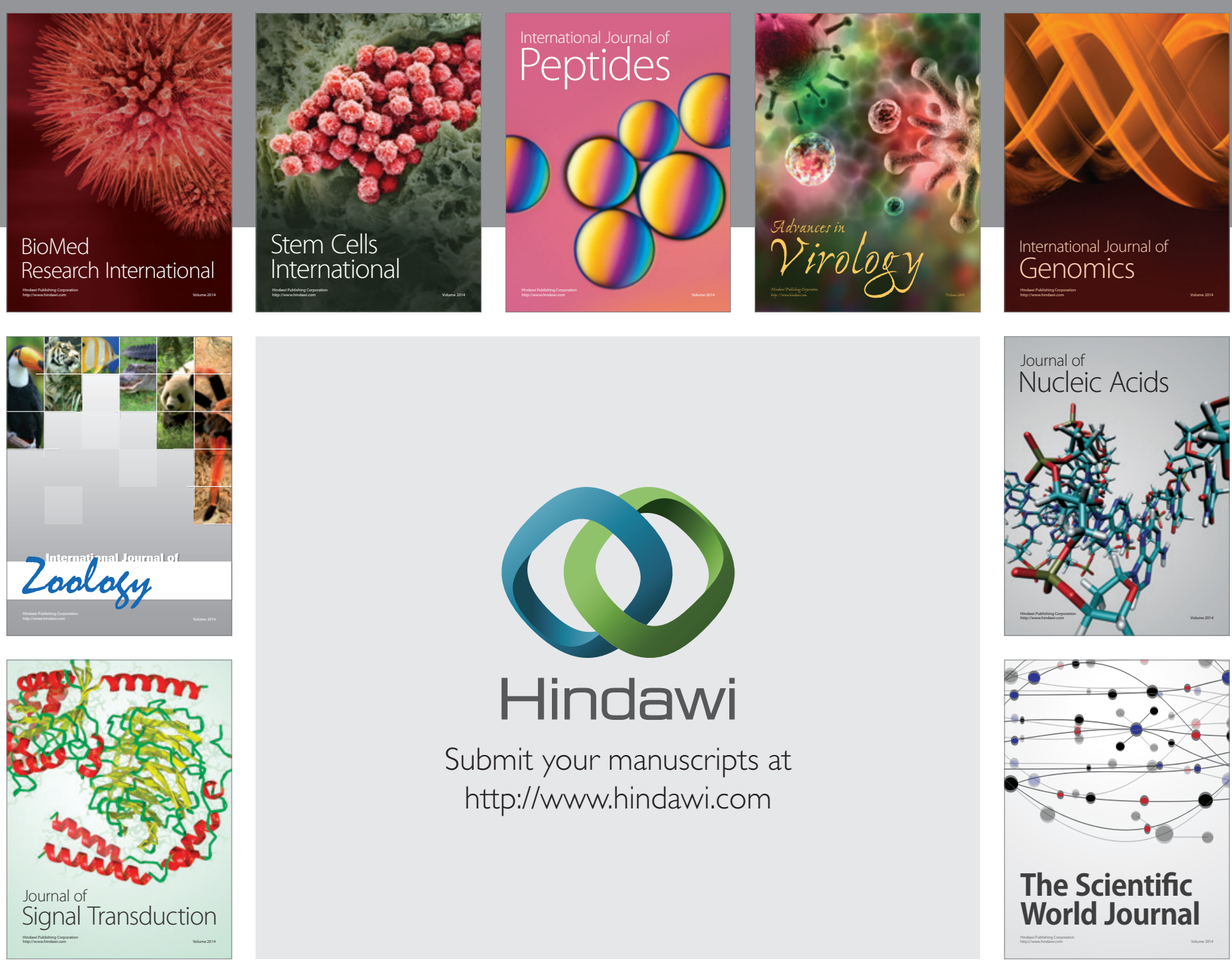

Submit your manuscripts at

http://www.hindawi.com
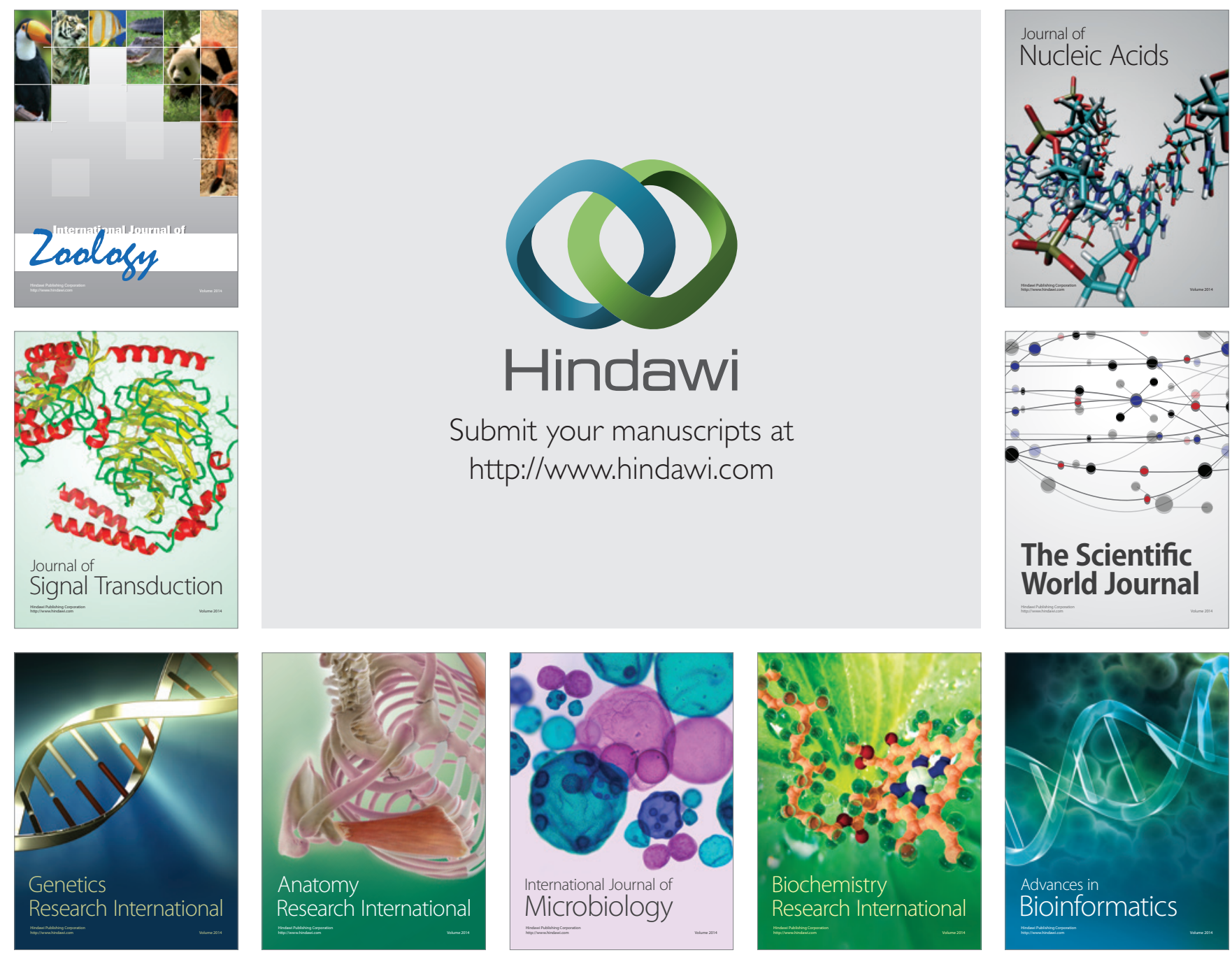

The Scientific World Journal
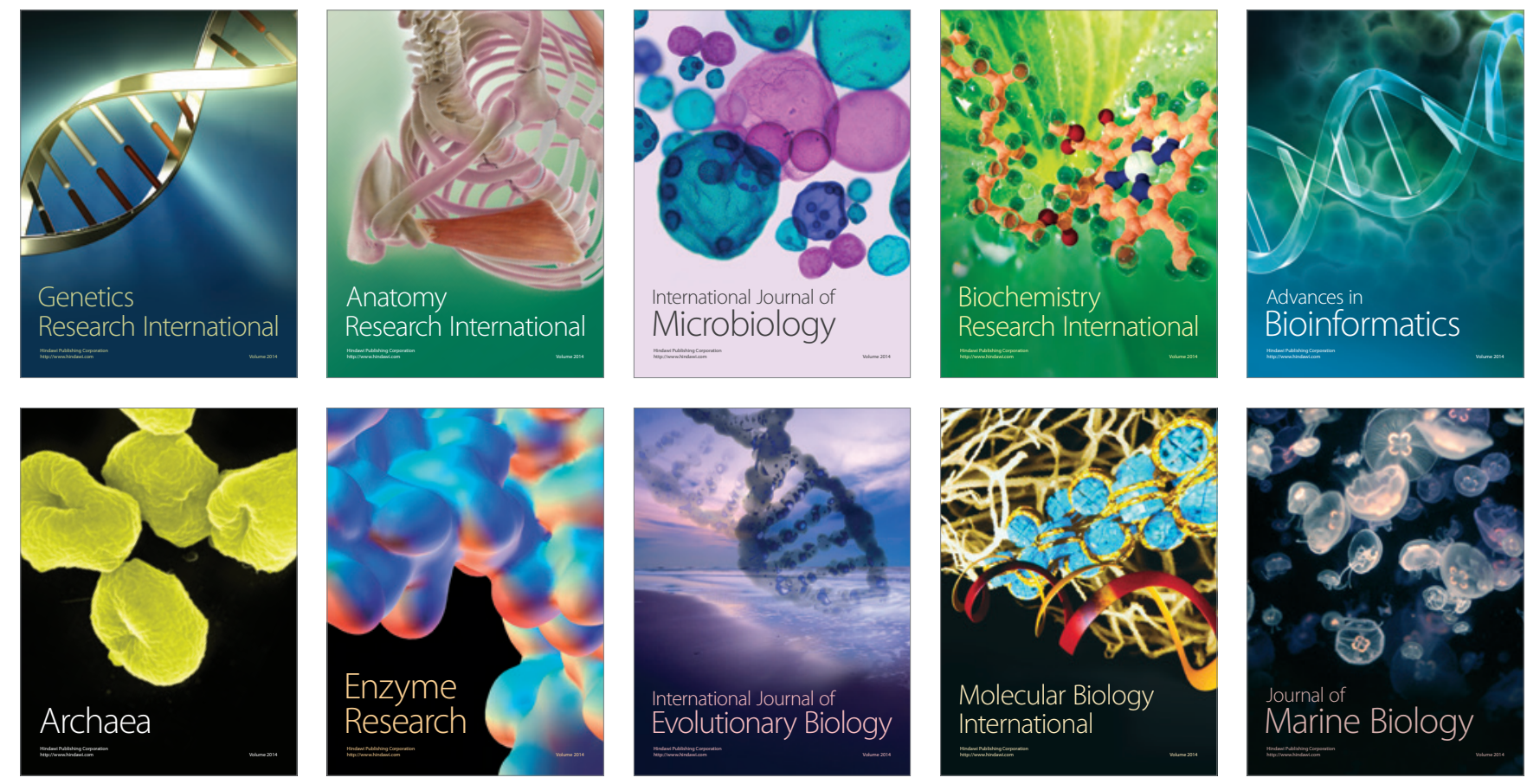